\title{
MODELO DE INTERAÇÃO GENÓTIPO × AMBIENTE PARA DADOS DE ENSAIOS MULTIAMBIENTAIS
}

Kuang Hongyu ${ }^{1}$

Fabiane de Lima Silva²

\section{INTRODUÇÃO}

O trigo é um dos três grãos mais produzidos globalmente, com milho e arroz. Em 2013, a produção mundial foi de 713 milhões de toneladas, ou seja, ficou em terceiro lugar, depois de milho (1.016.000) e arroz (745 milhões) (FAO, 2006). E foi o mais consumido por populações ocidentais desde os tempos antigos. $\mathrm{O}$ grão de trigo é usado para fazer farinha, integral, semolina, cerveja e uma variedade de produtos alimentares. O trigo híbrido tem tido sucesso limitado na Europa (especialmente na França), no sucesso comercial dos Estados Unidos e África do Sul (SHEWRY, HALFORD, 2002).

No momento em que surgem novas cultivares faz-se necessária a verificação do comportamento em novos ambientes, pois pode existir um comportamento diferencial das cultivares frente às variações ambientais denominado de interação genótipos $x$ ambientes $(G \times E)$ e o trigo também sofre estes efeitos, os quais podem dificultar a seleção de genótipos com adaptação ampla ou estáveis (YOKOMIZO et al., 2016).

A resposta diferencial de genótipos em ambientes é frequente em estudos de experimentos multiambientes (MET) e é conhecido como interação genótipo por ambiente $(G \times E)$, que reduz a correlação entre os valores fenotípicos e genotípicos e dificulta a seleção e recomendação de genótipos adaptados e estáveis (GAUCH, 2013; HONGYU et al., 2015).

1 Departamento de Estatística. Universidade Federal de Mato Grosso, Av. Fernando Corrêa da Costa, no 2367, Bairro Boa Esperança. CEP: 78060-900, Cuiabá, MT, Brasil. Email:prof.kuang@gmail.com

2 Centro de Ciências Agrárias, Ambientais e Biológicas - Universidade Federal do Recôncavo da Bahia. E-mail: fabianezte@yahoo.com.br 
A G $\times$ E ocorre em várias formas, com a forma mais extrema que consiste em interações cruzada, na classificação de genótipos mudanças entre ambientes, por exemplo, um genótipo responde de maneira diferente às variações ambientais e nesta situação, os melhores genótipos em um local podem não ser necessariamente os melhores em outros locais (HONGYU et al., 2015).

$A \mathrm{G} \times \mathrm{E}$ tem sido um foco de pesquisa entre biometristas e geneticistas quantitativos desde o início do século XX (YAN; KANG, 2003). Com a noção de que a $G \times E$ é indesejável e/ou que confunde avaliação de genótipos, muitos trabalhos têm se dedicado ao desenvolvimento de índices de estabilidade para quantificar e selecionar contra G × E (HONGYU et al., 2015).

Diversos métodos estatísticos destinados à avaliação da $\mathrm{G} \times$ E estão disponíveis no sentido de entender melhor este efeito e a escolha do método mais adequado depende dos dados experimentais, os estudos de $\mathrm{G} \times \mathrm{E}$ vêm se destacando e ganhando grande aplicabilidade nas duas últimas décadas (GAUCH, 2013; HONGYU, et al., 2014; RODRIGUES et al., 2014; HONGYU et al., 2015; YOKOMIZO et al., 2016).

Dentre as metodologias mais recentes, têm-se GGE biplot, que é um método baseado na análise de componentes principais para explorar os ensaios multiambientais e é permitido as visualizações dos gráficos de biplot as relações entre os ambientes de teste, genótipos e interação genótipo por ambiente (YAN; KANG, 2003).

O objetivo deste trabalho foi utilizar a técnica da análise de GGE biplot para investigação de mega-ambiente, avaliar a adaptabilidade e estabilidade genotípica nos dados de trigo multiambientes.

\section{DESENHO EXPERIMENTAL}

Foi utilizado neste trabalho o conjunto de dados MET sobre avaliação de produtividade de trigo provenientes pelo CIMMYT (INTERNATIONAZ MAIZE AND WHEAT IMPROVEMENT CENTER) em experimentos realizados em 13 países, com 20 genótipos de trigo, cada genótipo foi avaliado em 13 ambientes com 2 repetições no ano de 2005. O Ensaio de rendimento de trigo semi-árido (SAWYT) é um ensaio de rendimento replicado que contém germoplasma de trigo com pão de primavera (Triticum aestivum), adaptado a baixos 
períodos de precipitação, ambientes propensos a seca tipicamente recebendo menos de $500 \mathrm{~mm}$ de água disponível durante o ciclo de cultivo.

Todas as análises deste trabalho foram feitas por meio de rotinas computacionais implementadas no software R (R Development Core Team, 2014).

O modelo GGE biplot (YAN; KANG, 2003; YAN, 2011), que considera o efeito principal de genótipo mais a $G \times E$, são baseadas em gráficos biplot, representa graficamente uma matriz de dados. Este biplot (GABRIEL, 1971) é construído nos dois primeiros componentes principais de uma Análise de Componentes Principais (ACP) utilizando Modelos de Regressões Locais (SREG). O primeiro componente, quando se encontra altamente correlacionada com o efeito principal do genótipo, representa a proporção do rendimento que se deve somente às características do genótipo. $O$ segundo componente representa a parte do rendimento devida a $\mathrm{G} \times \mathrm{E}$ (YAN et al., 2000; YAN, 2011).

Quando diferentes cultivares estão adaptadas os diferentes grupos de ambientes e a variação entre grupos é maior do que dentro do grupo, tem-se a formação de um mega-ambiente (YAN; KANG, 2003). A definição de mega-ambientes e a relação entre os ambientes auxiliam os melhoristas de plantas na identificação de genótipos que possuam adaptação ampla ou específica a determinados ambientes ou grupos de ambientes (SILVA; BENIN, 2012). Na análise GGE biplot, quando se estuda mega-ambientes, a média no gráfico não está relacionada à média geral, mas sim à média do mega-ambiente e esta abordagem auxilia na identificação de genótipos que possuam adaptação ampla ou específica a determinados ambientes ou grupos de ambientes (YAN; KANG, 2003; YAN; TINKER, 2006).

Para um conjunto de dados MET, cada valor na tabela é a produtividade média de um genótipo em um ambiente (), que é a soma da média geral (), o efeito principal do genótipo () para o ambiente particular (), e a interação específica $(G \times E)$ entre o genótipo e o ambiente (), ignorando quaisquer erros aleatórios (YAN, 2011):

$$
y_{i j}=\mu+G_{i}+E_{j}+\phi_{i j}
$$


O modelo GGE biplot não separa os efeitos do genótipo e da $\mathrm{G} \times \mathrm{E}$, mantendo-os juntos em dois termos multiplicativos, que podem ser visualizados na equação (1) da metodologia SREG. Desde que apenas o G e G × E são pertinentes à avaliação genótipo, avaliação ambiente de teste, e delineamento de mega-ambiente; o efeito principal do ambiente $\mathrm{E}$ e a média geral devem ser removidas de cada elemento para apenas manter $\mathrm{G}$ e $\mathrm{G} \times \mathrm{E}$ na tabela de dupla entrada (YAN, 2002; YAN, 2011):

$$
y_{i j}-\mu-E_{j}=G_{i}+\phi_{i j}
$$

Os dados MET ambiente-centrado, após dimensionamento apropriado dos dados, são submetidas a decomposição em valores singulares e análise de biplot (YAN, 2011).

Yan e Tinker (2006) propôs uma "relação de informação (IR)" para avaliar a adequação de um biplot em exibir os padrões de uma tabela de dupla entrada. Suponha que esta tabela de dupla entrada tem $g$ genótipos e $e$ ambientes. $O$ número máximo de Componentes Principais (CPs) é requerido para representar completamente esta tabela é $k=\min (e, g$-1). Se não há correlação entre os ambientes, todos os $k$ CPs devem ser completamente independentes e a proporção da variação total explicada por cada CP deve ser exatamente $1 / k$.

Quando existir alguma correlação entre os ambientes, a proporção da variação explicada pelos os primeiros CPs deve ser maior do que $1 / k$, e a variação explicada por outros CPs deve ser inferior ou igual a 1/k (YAN; TINKER, 2006; YAN, 2011). A IR pode ser calculado para cada $C P$, que é a proporção da variação total explicada por cada PC multiplicado por $k$. A interpretação é a seguinte: um $\mathrm{CP}$ com IR $\geq 1$ contém padrões (associações entre ambientes), e um $\mathrm{CP}$ com IR $<1$ não contém qualquer padrão ou informação. $\mathrm{O}$ biplot de dimensão 2 representa adequadamente os padrões nos dados, se apenas os dois primeiros PCs têm um IR $\geq 1$ (YAN; TINKER, 2006; HONGYU et al., 2015). 


\section{RESULTADOS E DISCUSSÃO}

A interpretação dos gráficos GGE biplot permite avaliar a estabilidade dos genótipos nos ambientes por meio da observação da magnitude e do sinal dos escores dos genótipos e ambientes para o eixo da interação (Tabela 1). Portanto, os escores baixos (próximos de zero) representam os genótipos e ambientes mais estáveis (YAN; KANG, 2003; YAN, 2011). Na Tabela 1, o genótipo G5 apresentou escores mais próximos do valor zero, logo, é o genótipo mais estável neste conjunto de dados.

\begin{tabular}{|c|c|c|c|c|c|}
\hline \multirow[t]{2}{*}{ Genótipos } & \multicolumn{2}{|c|}{ GGE biplot } & \multirow[t]{2}{*}{ Ambientes } & \multicolumn{2}{|c|}{ GGE biplot } \\
\hline & $\mathrm{CP} 1$ & $\mathrm{CP} 2$ & & $\mathrm{CP} 1$ & $\mathrm{CP} 2$ \\
\hline G1 & $-0,73$ & $-1,59$ & $\mathrm{~A} 1$ & $-5,12$ & $-3,03$ \\
\hline $\mathrm{G} 2$ & 2,32 & 0,50 & $\mathrm{~A} 2$ & $-1,59$ & $-0,53$ \\
\hline G3 & $-0,93$ & 0,95 & A3 & $-2,84$ & $-1,44$ \\
\hline G4 & 0,34 & 1,65 & A4 & $-0,78$ & 2,16 \\
\hline G5 & $-0,01$ & $-0,23$ & A5 & $-0,4$ & $-0,55$ \\
\hline G6 & 2,99 & 1,10 & A6 & $-3,40$ & 2,87 \\
\hline G7 & 1,97 & $-0,41$ & A7 & $-0,22$ & 1,40 \\
\hline G8 & 1,06 & 0,22 & A8 & $-1,68$ & $-0,10$ \\
\hline G9 & $-2,29$ & 0,64 & A9 & $-2,46$ & 1,30 \\
\hline G10 & $-0,03$ & 3,36 & A10 & $-0,22$ & $-0,27$ \\
\hline G11 & $-0,42$ & 1,70 & A11 & $-3,23$ & $-0,20$ \\
\hline G12 & 0,06 & $-1,44$ & $\mathrm{~A} 12$ & $-2,27$ & 3,09 \\
\hline G13 & $-1,63$ & 0,05 & A13 & 0,53 & 0,80 \\
\hline G14 & $-1,17$ & $-0,52$ & & & \\
\hline G15 & 2,33 & $-2,16$ & & & \\
\hline G16 & 0,40 & $-2,52$ & & & \\
\hline G17 & $-2,21$ & $-0,95$ & & & \\
\hline G18 & $-1,15$ & $-2,76$ & & & \\
\hline G19 & 1,13 & 0,91 & & & \\
\hline $\mathrm{G} 20$ & $-2,03$ & 1,52 & & & \\
\hline
\end{tabular}

Tabela 1: Coordenadas dos eixos das abscissas (CP1) e das coordenadas (CP2) para os gráficos de GGE biplot

$\mathrm{Na}$ Tabela 2 os valores singulares dos 13 componentes principais (CPs), as variações explicitadas e a relação de informação (IR). 
Os valores de IR, apenas os três primeiros $P$ Cs contêm padrões ( $I R_{1}$ $=5,92>1 ; I_{2}=3,10>1$ e $I R_{3}=1,14>1$ ), o CP1 explicou 45,51\% da variação da interação, $23,81 \%$ para CP2 e 8,79\% para CP3. Os três componentes explicitaram total de $78,11 \%$ da interação, mas como os dois primeiros componentes são os mais importantes (YAN, 2011), portanto, o biplot (CP1 e CP2) é considerado adequado para representar os padrões dos dados. O GGE biplot com base neste conjunto de dados é apresentado na Figura 1, na abscissa do biplot apresentam-se os escores de CP1 e na ordenada os escores do CP2, dos genótipos e ambientes.

\begin{tabular}{cccc}
\hline CP & Valor singular & $\begin{array}{c}\text { Variação } \\
\text { Explicado (\%) }\end{array}$ & IR \\
\hline 1 & 8,60 & 45,51 & 5,92 \\
2 & 6,22 & 23,81 & 3,10 \\
3 & 3,78 & 8,79 & 1,14 \\
4 & 2,99 & 5,49 & 0,71 \\
5 & 2,67 & 4,38 & 0,57 \\
6 & 2,61 & 4,22 & 0,55 \\
7 & 2,10 & 2,74 & 0,35 \\
8 & 1,82 & 2,06 & 0,27 \\
9 & 1,45 & 1,29 & 0,17 \\
10 & 1,13 & 0,79 & 0,10 \\
11 & 0,89 & 0,49 & 0,06 \\
12 & 0,70 & 0,33 & 0,04 \\
13 & 0,59 & 0,10 & 0,03 \\
\hline
\end{tabular}

Tabela 2: Valor singular, proporção explicada e relação da informação (IR) dos quatro componentes principais (CPs)

A Análise de mega-ambiente foi feito baseado em GGE biplot da Figura 1. Os vértices do polígono são formados pelos genótipos: G10, G20, G9, G17, G18, G16, G15 e G6. Os 20 genótipos foram formados por 8 grupos pelas linhas que saíram da origem do biplot, também foram formados os 4 mega-ambientes: A4, A7, A12 e A13; A6 e A9; $A 2, A 3, A 8$ e $A 11 ; A 5$ e $A 10$ (Figura 1). 


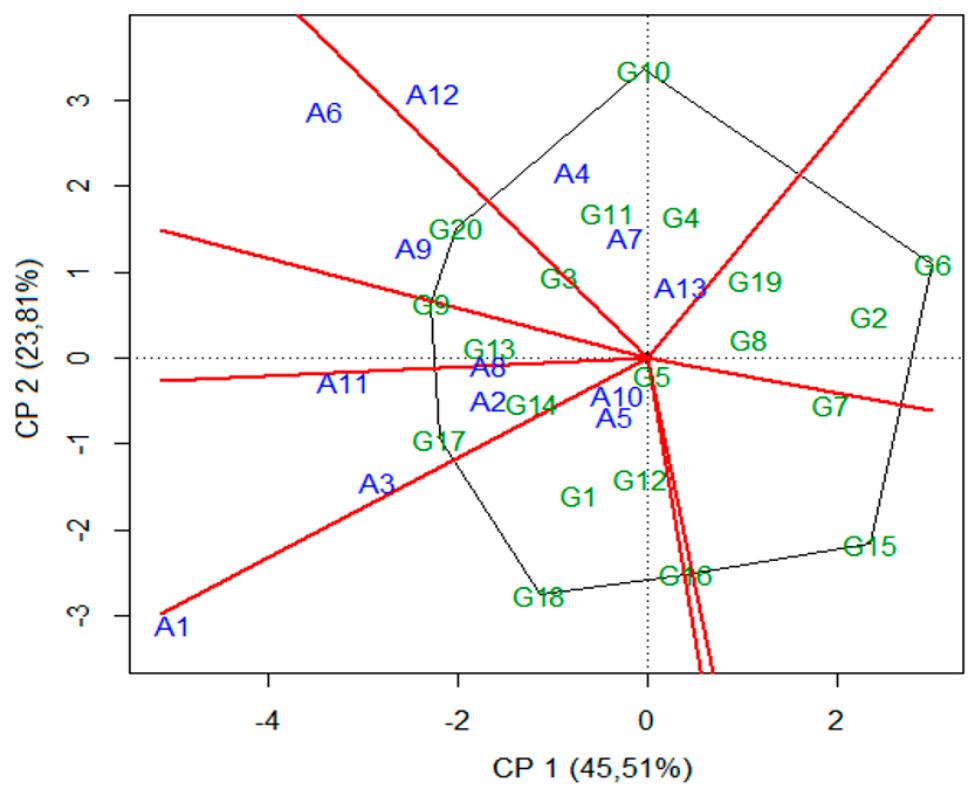

Figura 1 - O GGE biplot para análise de mega-ambiente nos dados de produtividade de trigo ( $\mathrm{t} / \mathrm{ha}$ )

O genótipo $\mathrm{G} 10$ é o vértice do setor em que o mega-ambiente formado pelos ambientes A4, A7, A12 e A13, portanto, é o genótipo que apresentou melhor desempenho nestes ambientes; o G20 é o vértice no setor em que o mega-ambiente formado por A6 e A9, logo, é o genótipo mais adaptado nestes ambientes; o G17 é o genótipo mais produtivo no mega-ambiente formado por $A 2, A 3$, A8 e A11 que o mesmo caso com o genótipo G18 para os ambientes A5 e A10 (Figura 1). Nos outros três setores formados com os vértices G6, G15 e G16 que não contêm ambientes, significa que estes genótipos não tem uma adaptação específica nos ambientes, que não apresentaram produção alta nos ambientes (Figura 1).

Um "ideótipo" é uma forma ideal de planta ou genótipo para determinado ambiente e objetivo de cultivo, portanto, o ideótipo é um genótipo que apresenta alto desempenho médio e alta estabilidade por meio de um mega-ambiente (HONGYU et al., 2015). A visualização do GGE biplot "Média versus Estabilidade" é uma ferramenta eficaz para a avaliação de genótipos em ambos os aspectos (YAN et al., 2007; YAN, 2011). Na figura 2, o pequeno círculo 
representa o "ambiente-média" e é definido pelas coordenadas médias de todos os ambientes de teste no biplot.

A linha reta com uma única seta que passa pela origem do biplot e do ambiente-média é referido como o "eixo do ambiente-média" ou EAM. A seta aponta para um maior desempenho médio para os genótipos. A linha com duas setas que passa pela origem do biplot e é perpendicular ao EAM, com as setas apontam para a maior variabilidade de desempenho (menor estabilidade) em ambas as direções (YAN, 2011; HONGYU et al., 2015).

A produtividade e estabilidade são os pontos mais importante para os melhoristas, na Figura 2 é possível identificar a questão de produtividade dos genótipos pelo EAM, os genótipos são classificados de acordo com sua produtividade média da seguinte forma: $\mathrm{G} 9>\mathrm{G} 20>\mathrm{G} 17>\mathrm{G} 13>\mathrm{G} 14>\mathrm{G} 10>\mathrm{G} 11>\ldots>\mathrm{G} 4>$ média geral $>$ $\mathrm{G} 5>\mathrm{G} 12>\ldots>\mathrm{G} 7>\mathrm{G} 6>\mathrm{G} 15$.

A reta perpendicular ao EAM foi utilizada para avaliar a questão de estabilidade dos genótipos, o G10 foi altamente instável, pois apresentaram produtividade baixa nos ambientes $A 1, A 3$ e A11, mas tem uma adaptação específica nos ambientes $A 4, A 7$ e A12; O G18 e G16 também foram instáveis, com rendimentos baixos principalmente nos ambientes A4, A6 e A12, com uma adaptação específica nos ambientes $A 5$ e A10. Os genótipos mais estáveis foram os G9, G13, G5 e G7, que não sofreram com o efeito da interação (Figura 2). Note-se que, se o biplot explica apenas uma proporção da variação total, alguns genótipos aparentemente estáveis podem não ser verdadeiramente estáveis como as suas variações não podem ser completamente explicadas neste biplot (YAN, 2011; HONGYU et al., 2015). 


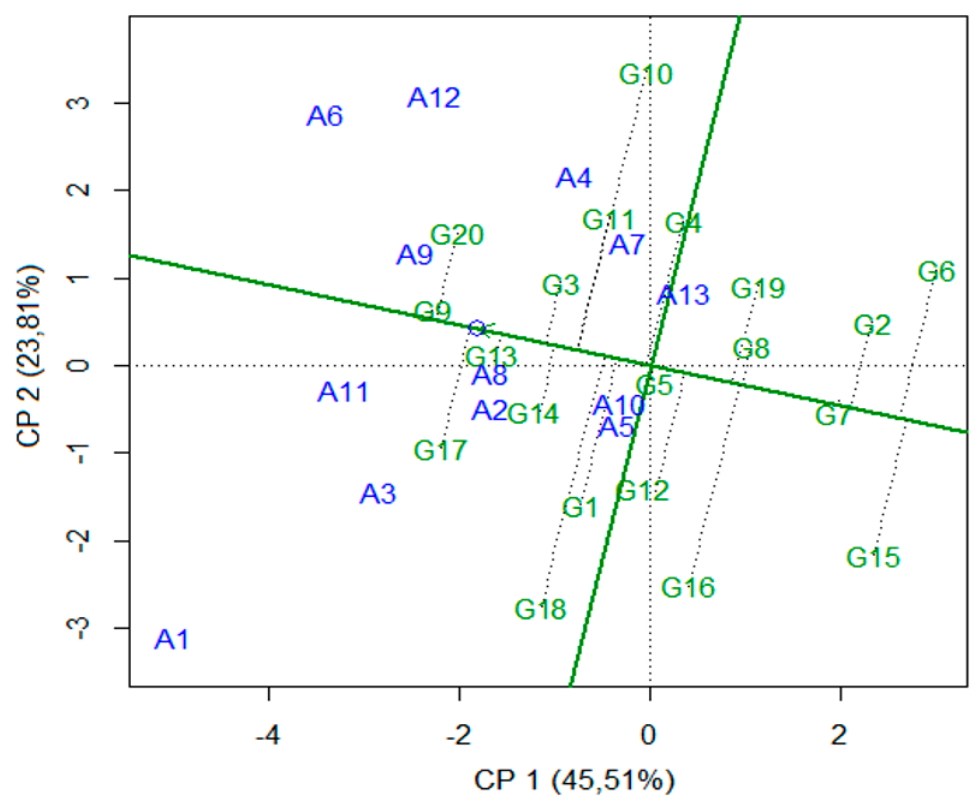

Figura 2: O GGE biplot ("Média versus Estabilidade") com eixo do ambiente-média (EAM) para mostrar o desempenho médio e estabilidade dos genótipos

Um ideótipo pode ser encontrado na Figura 3, como um ponto (centro dos círculos concêntricos) sobre o EAM, no sentido positivo e tem um comprimento do vetor igual aos vetores mais longos dos genótipos sobre o lado positivo do EAM, isto é, mais alto desempenho médio e estável. Portanto, os genótipos localizados mais perto do centro dos círculos concêntricos são mais desejáveis do que outros, e também são esses genótipos que procuram pelos melhoristas. Assim, G9 foi o ideótipo neste conjunto de dados, em seguida, os melhores foram os genótipos G13, G20, G3 e G14 (Figura 3). 


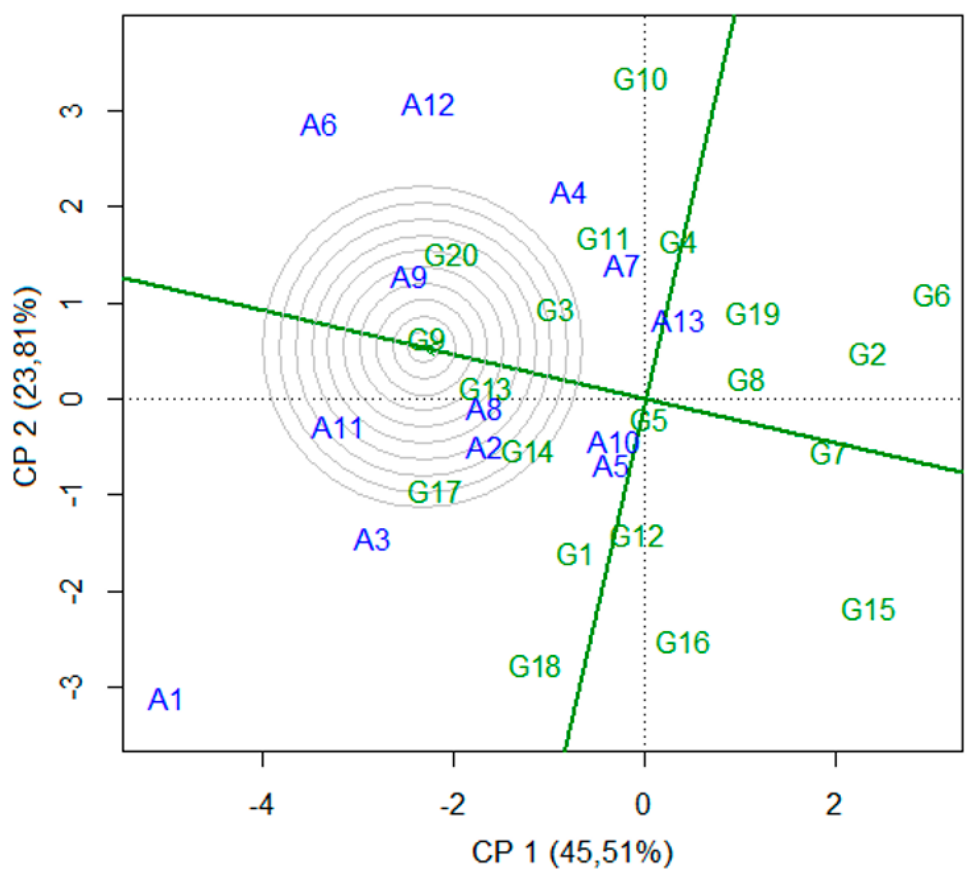

Figura 3: O GGE biplot com eixo do ambiente-média (EAM) para classificar os genótipos em relação ao ideótipo (no centro dos círculos concêntricos)

Os ambientes mais estáveis foram os ambientes A8, A2 A9 e $A 11$, foram os que menos sofreram pelo efeito da interação. $O$ ambiente $A 1$ foi o mais instável, mostrou falta de adaptação principalmente com os genótipos G6, G2, G10 e G11 (Figura 3).

A Figura 3 ilustra um conceito importante em relação à estabilidade, o termo "estabilidade elevada" só tem sentido quando associado ao desempenho médio; o genótipo estável é desejado apenas quando apresenta alta performance média (YAN; TINKER, 2006; YAN, 2011).

O número de eixos principais retidos para explicar e representar graficamente o padrão de interação G×E é variável, porém nos estudos tem-se observado que dois eixos são suficientes, o que foi observado por Hongyu et al. (2015) com os dois primeiros CPs capturando 63,96\% da variação total da interação, assim como para Baiyeri et al. (2000), com o primeiro capturando cerca de $55 \%$ e o segundo 35\%. De acordo com Tenkouano e Baiyeri (2007) dois 
eixos foram suficientes para explicar $88,3 \%$ da variação existente, também para Yokomizo et al. (2016) com os dois eixos foram suficientes e explicaram 89,01\% da interação.

\section{CONSIDERAÇÕES FINAIS}

A utilização da GGE biplot tornam mais eficazes a exploração da interação genótipo $\times$ ambiente e efetivamente resolveram os principais objetivos para os dados multiambientais.

A análise GGE biplot é uma técnica muito importante para melhoramento de plantas e na pesquisa agrícola. A análise fornece uma solução fácil e abrangente para o genótipo pela análise de dados multiambientais, que tem sido um desafio para melhoramento de plantas, geneticistas e agrônomos.

As análises GGE biplot podem ajudar a entender os ambientes de testes como um todo, ou seja, se consiste em mega-ambientes únicos ou múltiplos, o que determina se $\mathrm{G} \times \mathrm{E}$ pode ser explorada ou evitada. Na análise de mega-ambiente, GGE biplot pode ajudar a entender os ambientes sejam informativos, representativos e únicos em termos de discriminação de genótipos. Ao mesmo tempo pode ajudar a avaliar os genótipos em termos de desempenho médio e estabilidade em ambientes.

\section{REFERÊNCIAS}

BAIYERI, K. P.; MBAH, B. N.; TENKOUANO, A. Yield components of triploid and tetraploid Musa genotypes in Nigeria. Hort. Science, v. 35, p. 1338-1343, 2000.

FAO. Todas las estadísticas de producción mundial se basan en los datos oficiales de la. (Food and agriculture organization of the United Nations), 2006.

GABRIEL, K.R. The biplot graphic display of matrices with application to principal component analysis. Biometrika, v.58, n.3, p.453-467, 1971.

GAUCH, H. G. A Simple Protocol for AMMI Analysis of Yield Trials. Crop Science, v. 53, n.5, p.1860-1869, 2013.

HONGYU, K.; GARCIA-PEÑA, M.; ARAÚJO, L.B.; DIAS, C.T.S. Statistical analysis of yield trials by $A M M I$ analysis of genotype $x$ environment interaction. Biometrical Letters, Poznan, v.51, p.89-102, 2014.

HONGYU, K.; SILVA, F.L.; OLIVEIRA, A.C.S.; SARTI, D.A.; ARAÚJO, L.B.; DIAS, C.T.S. Comparação entre os modelos ammi e gge biplot para os dados de ensaios multi-ambientais. Rev. Bras. Biom., São Paulo, v.33, n.2, p.139-155, 2015. 
R DEVELOPMENT CORE TEAM. R: a language and environment for statistical computing. Vienna: R Foundation for Statistical Computing, Vienna, 2014.

RODRIGUES, P.C.; MALOSETTI, M.; GAUCH, H. G.; VAN EEUWIJK F.A. A weighted AMMI algorithm to study genotype-by-environment interaction and QTL-byenvironment interaction. Crop Science, v. 54, n. 4, p. 1555-1570, 2014.

SHEWRY. P.R; HALFORD, N.G. Cereal seed storage proteins: structures, properties and role in grain utilization. J Exp Bot (Revisión) 53 (370): 947-58. 2002.

SILVA, R. R.; BENIN, G. Análises Biplot: conceitos, interpretações e aplicações. Ciência Rural, Santa Maria, v.42, n.8, p.1404-1412, 2012.

TENKOUANO, A.; BAIYERI, K. P. Adaptation pattern and yield stability of banana and plantain genotypes grown in contrasting agroecologies in Nigeria. 8th African Crop Science Society Conference, El-Minia, Egypt, 27-31 October 2007. Proceedings, v. 8, p. 337-384, 2007.

YAN, W. Singular-value partition for biplot analysis of multi-environment trial data. Agronomy Journal, v. 94, n. 5, p. 990-996, 2002.

YAN, W. GGE Biplot vs. AMMI Graphs for Genotype-by-Environment Data Analysis. Journal of the India Society of Agricultural Statistics, v.65, n.2, p.181-193, 2011.

YAN, W.; KANG, M.S. GGE Biplot Analysis: A Graphical Tool for Breeders, Geneticists, and Agronomists. CRC Press, Boca Raton, FL, USA, 2003. 271 p.

YAN, W; TINKER, A. Biplot analysis of multi-environment trial data: principles and applications. Canadian Journal of Plant Science, v.86, n.3, p.623-645, 2006.

YAN, W.; HUNT, L.A.; SHENG, Q.; SZLAVNICS, Z. Cultivar evaluation and megaenvironment investigation based on GGE biplot. Crop Science, v.40, n. 3, p.597605, 2000.

YAN, W.; KANG, M.S.; MA, B.; WOODS, S.; CORNELIUS, P.L. GGE biplot vs. AMMI analysis of genotype-by-environment data. Crop Science, v.47, n.2, p.643-653, 2007.

YOKOMIZO, G.K.; DIAS, J.S.A.; DIAS, C.T.S.; HONGYU, K. Análise AMMI em caracteres vegetativos de genótipos de bananeiras no Amapá. Rev. Cienc. Agrar., v. 59, n. 1, p. 1-8, jan./mar. 2016. 